\title{
ON EVENTUALLY EXPANDING MAPS OF THE INTERVAL
}

\author{
PEYMAN ESLAMI AND PAWEL GORA
}

\begin{abstract}
In this paper we conjecture that the piecewise linear map $f(x)=p x \mathbb{I}_{[0,1 / p)}(x)+$ $(s x-s / p) \mathbb{I}_{[1 / p, 1]}(x), p>1,0<s<1$ which has an expanding, onto branch and a contracting branch is eventually piecewise expanding. We give a partial proof of the conjecture, in particular for values of $p$ and $s$ such that $\left\lceil-\frac{\ln (p(1-s)+s)}{\ln s}\right\rceil \neq\left\lceil-\frac{\ln p}{\ln s}\right\rceil$.
\end{abstract}

\section{INTRODUCTION}

A piecewise differentiable function $f$ is expanding if $\left|f^{\prime}(x)\right|>1$ for all $x$ at which the derivative exists. $f$ is said to be eventually expanding if there exists $N \in \mathbb{N}$ such that $f^{N}(x)$ (the $N$-fold composition of $f$ with itself) is expanding.

Eventually expanding maps play an important role in dynamical systems theory. For example, most theorems on existence of absolutely continuous invariant measures require the map to be expanding or eventually expanding. Very often proofs for general maps are reduced to the eventually expanding situation. However, showing that a map is eventually expanding is far from trivial. As a simple example, let $f$ be a piecewise linear function on the unit interval $[0,1]$ with two increasing branches, one of which has slope greater than one and the other less than one. This is one of the simplest maps one can make that is not expanding, but it seems to be rather difficult to show that it is eventually expanding. In this paper we conjecture that $f$ is eventually expanding if the first branch is onto, and the second branch is touching the $x$-axis. We provide a partial proof of the conjecture. This family of maps was investigated in [4] by different methods. For $p \leq 2$ ( $p$ being slope of the first branch) its natural extension was constructed and proved to be Bernoulli. Similar, but different type of maps were analyzed in [2] and [6] and shown to admit absolutely continuous invariant measures.

The authors recently learned about the main result of preprint [3], which covers a wider class of maps than those considered here.

\section{Main Theorem}

Let $f:[0,1] \rightarrow[0,1]$ be defined by

$$
f(x)=\left\{\begin{array}{ll}
p x, & 0 \leq x<\frac{1}{p} \\
s\left(x-\frac{1}{p}\right) & \frac{1}{p} \leq x \leq 1
\end{array} .\right.
$$

Figure 1 shows the graph of $f$ for $p=7 / 2$ and $s=1 / 2$.

Conjecture 1. For all $(s, p) \in(0,1) \times(1, \infty), f$ is eventually expanding.

For a real number $x$, let $\lceil x\rceil=\min \{n \in \mathbb{Z} \mid n \geq x\}$ and $\lfloor x\rfloor=\max \{n \in \mathbb{Z} \mid n \leq x\}$. 


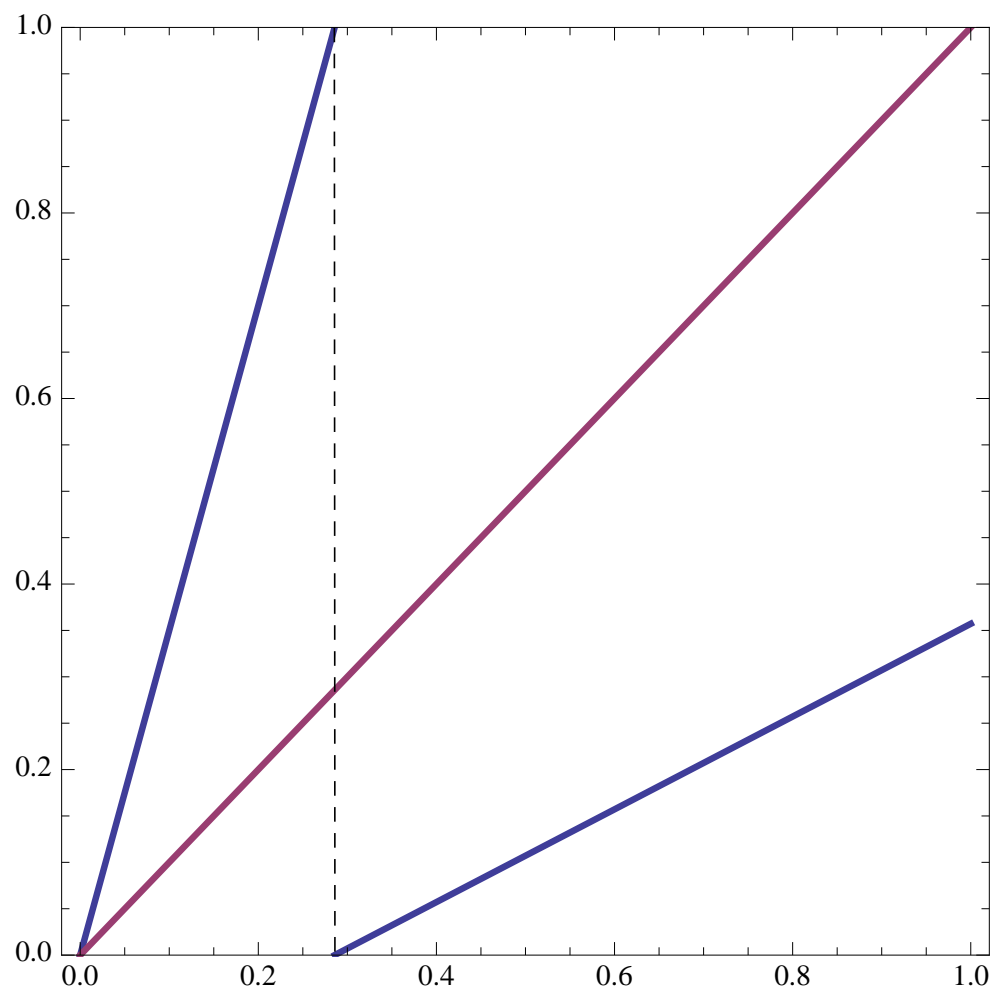

Figure 1 . Graphs of $y=x$, and $y=f(x)$ for $p=7 / 2$ and $s=1 / 2$.

Theorem 1. For all $(s, p) \in(0,1) \times(1, \infty)$ such that

$$
\left\lceil-\frac{\ln (p(1-s)+s)}{\ln s}\right\rceil \neq\left\lceil-\frac{\ln p}{\ln s}\right\rceil
$$

$f$ is eventually expanding.

Proof. Consider a positive integer $N$ and for any $x \in[0,1]$, consider the sequence $x, f(x), f^{2}(x), \ldots, f^{N-1}(x)$. There are only finitely many values of $x$ such that for some $i$ with $0 \leq i<N, f^{i}(x)=1 / p$. These finitely many values of $x$ divide the interval $[0,1]$ into finitely many intervals. If $J$ is one of these intervals, then one can verify by induction that for all $i<N, f^{i}(J)$ is an interval that does not contain $1 / p$, so it is contained in either $[0,1 / p)$, or $(1 / p, 1]$. If $f^{i}(J) \subset[0,1 / p)$, then for all $x \in J, f^{i+1}(x)$ is obtained from $f^{i}(x)$ by applying the first branch of $f$, and if $f^{i}(J) \subset(1 / p, 1]$ then $f^{i+1}(x)$ is obtained from $f^{i}(x)$ by applying the second branch of $f$. In the first case we say that $f$ is expanding on $J$ at step $i+1$ and in the second case we say that it is contracting. It is easy to see that $f^{N}$ is linear on $J$, with slope $p^{m} s^{n}$, where $m$ and $n$ are the numbers of steps at which $f$ is expanding and contracting on $J$. Note that $0 \leq n, m \leq N$ and $m+n=N$.

Let $A=\{(s, p):\lceil-\ln p / \ln s\rceil \neq\lceil-\ln (p(1-s)+s) / \ln s\rceil\}$. Suppose there exist $(s, p) \in A$ such that for every $N \in \mathbb{N}, f^{N}$ is not expanding. Then for every $N$ there exists an interval $J \subset I$, as described above, on which $f$ is linear with slope $p^{m} s^{n} \leq 1$. 
This implies

$$
m \leq c N, \text { where } c=\frac{-\ln s}{\ln p-\ln s} .
$$

$J$ expands $m$ times and contracts $n$ times during $N$ iterations, hence $J$ must contract consecutively $\lceil n /(m+1)\rceil$ times during $N$ iterations of $f$. That is, there exists $i \in \mathbb{N}$ such that $f^{i+k}(J) \subset(1 / p, 1]$, for $k=0,1, \ldots,\lceil n(m+1)\rceil-1$.

Since $m \leq c N$ and and $m$ is an integer, $m \leq\lfloor c N\rfloor$, and therefore

$$
\left\lceil\frac{n}{m+1}\right\rceil \geq\left\lceil\frac{N-\lfloor c N\rfloor}{\lfloor c N\rfloor+1}\right\rceil=\left\lceil\frac{\frac{1}{c}-\frac{\lfloor c N\rfloor}{c N}}{1+\frac{1-(c N)}{c N}}\right\rceil \geq\left\lceil\frac{\frac{1}{c}-1}{1+\epsilon_{N}}\right\rceil,
$$

where $(c N)$ denotes the fractional part of $c N$ and $\epsilon_{N}=(1-(c N)) / c N \geq 0$. Note that $\epsilon_{N} \rightarrow 0$ as $N \rightarrow \infty$. Therefore, there exists $N_{0} \in \mathbb{N}$ such that for every $N>N_{0}$, $\left\lceil(1 / c-1) /\left(1+\epsilon_{N}\right)\right\rceil=\lceil 1 / c-1\rceil=\lceil-\ln p / \ln s\rceil$. Taking $N=N_{0}+1$, we conclude that there is an interval $J$ that, in the first $N$ iterations of $f$, has $j=\lceil-\ln p / \ln s\rceil$ consecutive contractions. This means that there is some $i$ such that for all $x \in f^{i}(J)$, $f^{k}(x)>1 / p$ for $k=0,1, \ldots, j-1$. Letting $x$ be any point in the interval $f^{i}(J)$, we find that

$$
\frac{1}{p}<f^{j-1}(x)=s^{j-1} x-\frac{s}{p}\left(\frac{1-s^{j-1}}{1-s}\right) \leq s^{j-1}-\frac{s}{p}\left(\frac{1-s^{j-1}}{1-s}\right),
$$

which means that

$$
j-1<-\frac{\ln (p(1-s)+s)}{\ln s} .
$$

Since $j$ is an integer, this implies that

$$
\left\lceil-\frac{\ln (p(1-s)+s)}{\ln s}\right\rceil \geq j=\left\lceil-\frac{\ln p}{\ln s}\right\rceil .
$$

But since $0<s<1$ and $p>1, p(1-s)+s<p$, so

$$
\left\lceil-\frac{\ln (p(1-s)+s)}{\ln s}\right\rceil \leq\left\lceil-\frac{\ln p}{\ln s}\right\rceil
$$

and therefore

$$
\left\lceil-\frac{\ln (p(1-s)+s)}{\ln s}\right\rceil=\left\lceil-\frac{\ln p}{\ln s}\right\rceil
$$

Therefore, $(s, p) \notin A$, a contradiction.

The complement of set $A$ in the proof of theorem 1 is given by $\lceil-\ln p / \ln s\rceil=$ $\lceil-\ln (p(1-s)+s) / \ln s\rceil$. If we solve this equation for $p$, we get the regions

$$
1+\frac{1}{s}+\cdots+\frac{1}{s^{k}}<p \leq \frac{1}{s^{k+1}}, \text { for } k \in \mathbb{N} \cup\{0\} .
$$

Hence, the curves on the boundary of the region $A$ are of the form $1+\frac{1}{s}+\cdots+\frac{1}{s^{k}}$, and $\frac{1}{s^{k+1}}$. 


\section{OTHER REGIONS WHERE $f$ IS EVENTUALLY EXPANDING}

We will refer to $\{(s, p) \in(0,1) \times(1, \infty) \mid f$ is eventually expanding $\}$ as the "good" region. We show that the good region contains all points with small enough $p$ :

Proposition 1. If $1<p \leq 2$ and $0<s<1$, then $f$ is eventually expanding.

Proof. Let $N$ be the least positive integer such that $p^{N-1} s>1$. Note that $N \geq 2$ and $p^{N-2} s \leq 1$. Consider the first $N$ iterations of $f$. As in the proof of Theorem 1 , $f^{N}$ is piecewise linear on $[0,1]$, and if $J$ is one of the intervals on which $f$ is linear, then the slope of $f$ on $J$ is $p^{m} s^{n}$, where $m$ and $n$ are the numbers of expansions and contractions of $J$ under $f$. We claim now that we always have $n \leq 1$, so the slope is at least $p^{N-1} s>1$ and therefore $f$ is eventually expanding. To prove this claim, suppose that $n \geq 2$. Then there must be some $i$ and $j$ such that $0 \leq i<j \leq N-1$, $f$ is contracting on $J$ at steps $i+1$ and $j+1$ and $f$ is expanding on $J$ at step $k+1$ whenever $i<k<j$. In other words, $f^{i}(J) \subset(1 / p, 1], f^{j}(J) \subset(1 / p, 1]$, and if $i<k<j$ then $\left.f^{k}(J) \subset[0,1 / p)\right)$. This means that if $x \in f^{i}(J)$ then $x>1 / p, f^{k}(x)<1 / p$ for $k=1, \ldots, j-i-1$, and $f^{j-1}>1 / p$. But then

$$
f^{i-j}(x)=p^{i-j-1} s\left(x-\frac{1}{p}\right) \leq p^{N-2} s\left(x-\frac{1}{p}\right) \leq x-\frac{1}{p} \leq 1-\frac{1}{p}=\frac{p-1}{p} \leq \frac{1}{p},
$$

which is a contradiction.

Denote the boundary curves of the region $A$ by:

$$
\begin{aligned}
\gamma_{k}^{L}(s) & =\frac{1}{s^{k}}, \text { and } \\
\gamma_{k}^{U}(s) & =1+\frac{1}{s}+\frac{1}{s^{2}}+\cdots+\frac{1}{s^{k}}=\frac{1-s^{k+1}}{s^{k}(1-s)},
\end{aligned}
$$

where $k \in \mathbb{N} \cup\{0\}$. The following lemma shows that for $p \in\left[\gamma_{n-1}^{U}(s), \gamma_{n}^{U}(s)\right)$, the $n$-th image of 1 is the first image of 1 to fall into $[0,1 / p)$.

Lemma 1. For $n \geq 0, f^{j}(1) \geq 1 / p$ for $j=0,1, \ldots, n$ if and only if $p \geq \gamma_{n}^{U}(s)$. If $p=\gamma_{n}^{U}(s)$, then $f^{n}(1)=1 / p$.

Proof. We prove it by induction. If $n=0$, then the equivalence to be proven says that $1 \geq 1 / p$ if and only if $p \geq 1$ and that is clearly true. For the induction step, assume that the statement is true for $n=k-1$. Then to prove the statement for $n=k$ it suffices to show that for $p \geq \gamma_{k-1}^{U}(s), f^{k}(1) \geq 1 / p$ if and only if $p \geq \gamma_{k}^{U}(s)$. So suppose that $p \geq \gamma_{k-1}^{U}(s)$. Then by inductive hypothesis, $f^{i}(1) \geq 1 / p$ for $i=0, \ldots, k-1$. Therefore

$$
f^{k}(1)=s^{k}-\frac{s}{p}\left(\frac{1-s^{k}}{1-s}\right)
$$

and we have

$$
f^{k}(1) \geq \frac{1}{p} \Longleftrightarrow s^{k}-\frac{s}{p}\left(\frac{1-s^{k}}{1-s}\right) \geq \frac{1}{p} \Longleftrightarrow p \geq \frac{1}{s^{k}}\left(\frac{1-s^{k+1}}{1-s}\right)=\gamma_{k}^{U}(s) .
$$

Similar inductive argument shows $f^{n}(1)=1 / p$ if $p=\gamma_{n}^{U}(s)$. 
Consider any $s \in(0,1)$ and any $k \geq 2$. For $p \geq \gamma_{k-1}^{U}(s)$ we have

$$
f^{k}(1)=s^{k}-\frac{s}{p}\left(\frac{1-s^{k}}{1-s}\right),
$$

which clearly increases as $p$ increases. Also, if $p=\gamma_{k-1}^{U}(s)$ then $f^{k-1}(1)=1 / p$ and therefore $f^{k}(1)=0$, and if $p=\gamma_{k}^{U}(s)$, then $f^{k}(1)=1 / p$. It follows that there is a unique $p \in\left(\gamma_{k-1}^{U}(s), \gamma_{k}^{U}(s)\right)$ such that $f^{k}(1)=1 / p^{2}$. We denote this unique value of $p$ by $\gamma_{k}^{M}(s)$. Clearly if $\gamma_{k-1}^{U}(s) \leq p<\gamma_{k}^{M}(s)$ then $f^{k}(1)<1 / p^{2}$, and if $p>\gamma_{k}^{M}(s)$ then $f^{k}(1)>1 / p^{2}$. We can find a formula for $\gamma_{k}^{M}(s)$ by setting the formula for $f^{k}(1)$ above equal to $1 / p^{2}$ and solving the resulting quadratic equation we get

$$
\gamma_{k}^{M}(s)=\frac{1-s^{k}+\sqrt{\left(1-s^{k}\right)^{2}+4(1-s)^{2} s^{k-2}}}{2(1-s) s^{k-1}} .
$$

Proposition 2. Suppose $k \geq 2$. If $1 / 2<s<1$, and

$$
\gamma_{k-1}^{U}(s) \leq p \leq \gamma_{k}^{M}(s)=\frac{1-s^{k}+\sqrt{\left(1-s^{k}\right)^{2}+4(1-s)^{2} s^{k-2}}}{2(1-s) s^{k-1}},
$$

then $f$ is eventually expanding.

Proof. Let $N$ be a positive integer, and consider $N$ iterations of $f$. As usual, let $J$ be an interval on which $f^{N}$ is linear, with slope $p^{m} s^{n}$, where $m$ and $n$ are the numbers of expansions and contractions of $J$ in the $N$ iterations of $f$. Since $\gamma_{k-1}^{U}(s) \leq p<\gamma_{k}^{M}(s)$, we have $f^{k}(1) \leq 1 / p^{2}$. It follows that $J$ can never have more than $k$ consecutive contractions, and if it has $k$ consecutive contractions and those contractions are followed by at least two more steps, then both of those steps must be expansions.

The sequence of contractions and expansions of $J$ can be described by a string of $c$ 's and $e$ 's, where the $i$ th letter is a $c$ if $J$ contracts at step $i$ and an $e$ if it expands. This string can be broken up into blocks of the form $c^{i} e$, where $0 \leq i \leq k-1$, or $c^{k} e e$, except possibly for a final block consisting of up to $k c^{\prime}$ 's, perhaps followed by an $e$. If we associate with each block of the form $c^{i} e^{j}$ the factor $s^{i} p^{j}$, then the product of all of these factors is $p^{m} s^{n}$, the slope of $f^{N}$ on $J$.

For a block of the form $c^{i} e$ with $0 \leq i \leq k-1$, the corresponding factor is $s^{i} p \geq s^{k-1} p$, and for a block of the form $c^{k} e e$ the factor is $s^{k} p^{2}$. Since $p \geq \gamma_{k-1}^{U}(s)=1+\cdots+1 / s^{k-1}>$ $1 / s^{k-1}$, we have $s^{k-1} p>1$. And $s p \geq s^{k-1} p>1$, so $s^{k} p^{2}=(s p)\left(s^{k-1} p\right)>s^{k-1} p$. Thus for all blocks except the last, the factor is at least $s^{k-1} p$, which is greater than 1 . The factor for the last block is at least $s^{k}$. The length of the last block is at most $k+1$, and the length of every other block is at most $k+2$, so the number of blocks is at least $N /(k+2)$. Therefore the slope of $f^{N}$ on $J$ is at least

$$
\left(s^{k-1} p\right)^{N /(k+2)-1} s^{k} .
$$

Since $s^{k-1} p>1$, this will be larger than 1 for sufficiently large $N$, so $f$ is eventually expanding.

Corollary 1. If $1<p$ and $\frac{1}{2} \leq s<1$, then $f$ is eventually expanding. 
Proof. Suppose $f$ is not eventually expanding. Then by Theorem 1 , there is some $k \geq 1$ such that $\gamma_{k-1}^{U}(s)<p \leq \gamma_{k}^{L}(s)$. If $k=1$ then this means $1<p \leq 1 / s \leq 2$, contradicting Proposition 1. Now suppose $k \geq 2$. Since $p \leq \gamma_{k}^{L}(s)=1 / s^{k}$, we have $s^{k} \leq 1 / p$, and therefore

$$
f^{k}(1)=s^{k}-\frac{s}{p}\left(\frac{1-s^{k}}{1-s}\right) \leq \frac{1}{p}-\frac{s}{p}\left(\frac{1-1 / p}{1-s}\right)=\frac{1}{p^{2}}-\left(\frac{2 s-1}{1-s}\right)\left(\frac{p-1}{p^{2}}\right) \leq \frac{1}{p} .
$$

Therefore $p \leq \gamma_{k}^{M}(s)$, so we have a contradiction with Proposition 2 .

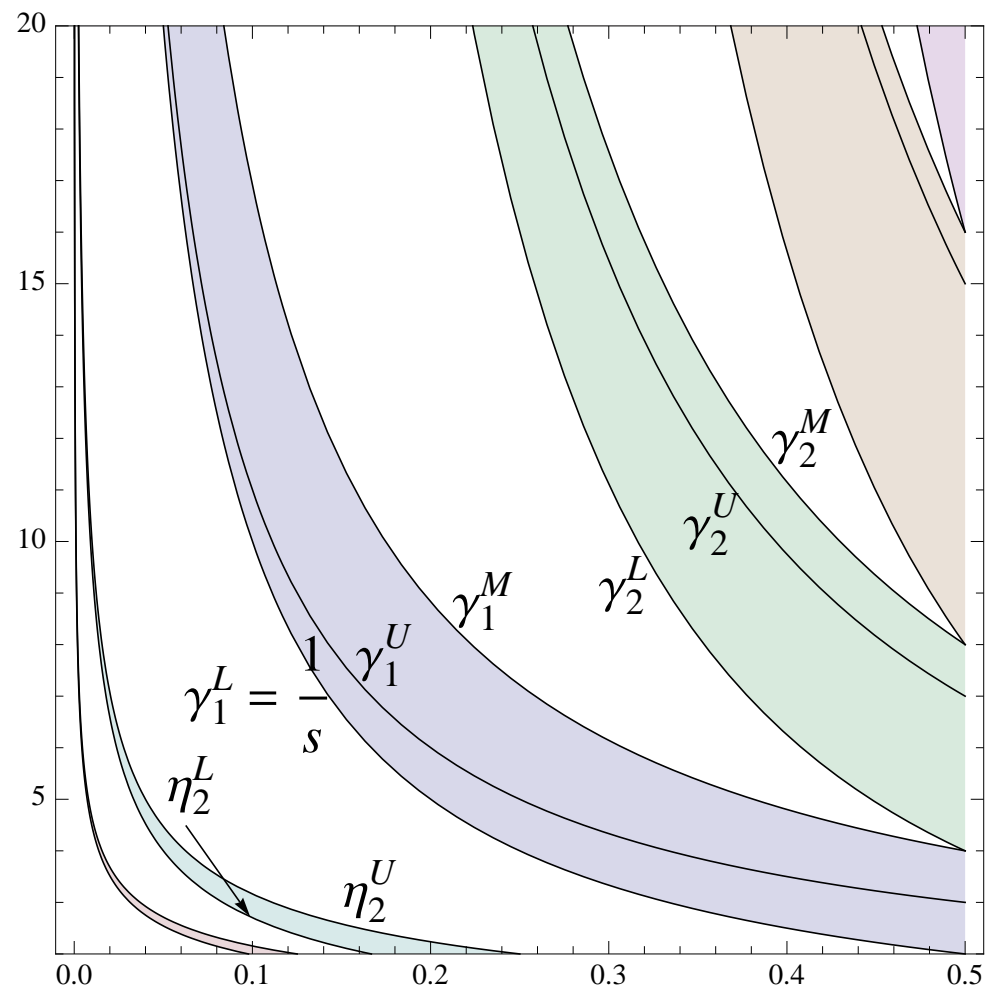

Figure 2. Shows the regions where $f$ is proven to be eventually expanding for $p>2$ and $s<1 / 2$. If $1<p \leq 2$ or $s \geq 1 / 2, f$ is eventually expanding by Proposition 1 and Corollary 1. It is conjectured that $f$ is eventually expanding for all $1<p$ and $0<s<1$.

The following proposition shows other parts of the good region.

Proposition 3. If $s p<1$ and $\frac{1}{p^{k}}<s<\frac{1}{p^{k-1}(p-1)}$, $k \geq 2$, then $f$ is eventually expanding.

Proof. If $p<1 / s$, then $f(1)=s(1-1 / p)<1 / p$. Also, if $s<\frac{1}{p^{k-1}(p-1)}$, then $f(1)=$ $s(1-1 / p)<1 / p^{k}$. It follows that in $k+1$ iterations, any interval can contract at most once. So on any of the intervals on which $f^{k+1}$ is linear, the slope is at least $s p^{k}>1$.

Therefore, if we let $\eta_{k}^{U}(p)=1 /\left(p^{k-1}(p-1)\right)$ and $\eta_{k}^{L}(p)=1 / p^{k}$ where $k \geq 2$, then for $(s, p)$ satisfying $\eta_{k}^{L}(p)<s<\eta_{k}^{U}(p), f$ is eventually expanding. 
Figure 2 shows the regions where we have proven $f$ to be eventually expanding (darker regions) for $p>2$ and $s<1 / 2$. Visible curves are $\gamma_{1}^{L}(p)=1 / s \leq \gamma_{1}^{U}$ (inside) $\leq \gamma_{2}^{M} \leq$ $\gamma_{2}^{L} \leq \gamma_{2}^{U}$ (inside) $\leq \gamma_{3}^{M}$, and $\eta_{3}^{L} \leq \eta_{3}^{U} \leq \eta_{2}^{L} \leq \eta_{2}^{U} \leq \gamma_{1}^{L}(p)=1 / s$. Note that $f$ is also eventually expanding If $1<p \leq 2$ or $s \geq 1 / 2$ as shown by Proposition 1 and Corollary 1.

\section{ExaCtNess AND OTHER PROPERTIES}

A function $f: I \rightarrow I$ is said to be exact or locally eventually onto, if for every open interval $J \in I$ there exists $N$ such that $f^{N}(J)=I$.

Proposition 4. The map $f$ defined by (1) is exact (or locally eventually onto) if it is eventually expanding.

Proof. By assumption, there exists $N$ such that $f^{N}$ is piecewise expanding. Since both branches of $f$ touch the $x$ axis, all branches of $f^{j}$ touch the $x$ axis, for any $j \in \mathbb{N}$. Since $f^{N}$ is piecewise expanding, any given interval $J \in[0,1]$ grows under action of $f^{N}$ until its image covers a discontinuity point. Thus, there exists an integer $k$ such that $f^{k N+1}(J)$ contains the fixed point 0 . Since the branch that contains the fixed point is onto and expanding, some iterate of $f^{k N+1}(J)$ under $f$ eventually covers all of $[0,1]$.

If map $f$ is eventually piecewise expanding, the whole rich theory of such maps applies to it. In particular $f$ admits an absolutely continuous invariant measure $\mu$ $[1,7]$. Similarly as in Proposition 4 it can be proven that $\mu$ is unique and the system $\{f, \mu\}$ is exact in measure theoretical sense. Explicit formula for the density of $\mu$ can be obtain using methods of [5].

A point $x$ is called periodic under $f$ if there exists $N \in \mathbb{N}$ such that $f^{N}(x)=x$. In this case, $x$ is said to be repelling if $\left|\left(f^{N}\right)^{\prime}(x)\right|>1$ and attracting if $\left|\left(f^{N}\right)^{\prime}(x)\right|<1$. The following property of $f$ has been noticed by M. Misiurewicz.

Proposition 5. (Misiurewicz) All periodic points of $f$ are repelling.

Proof. Let us fix an $N \geq 1$. All branches of $f^{N}$ are increasing and touch the $x$ axis. The slope of $f^{N}$ at 0 is $p^{N}>1$. Thus, no branch with a slope smaller than or equal to 1 can intersect the diagonal. Thus, any fixed point of $f^{N}$ is repelling.

\section{Acknowledgements}

We would like to thank Dr. Misiurewicz for his valuable remarks on this problem. We are also grateful to the reviewers and the editor for their helpful suggestions and corrections.

\section{REFERENCES}

1. Boyarsky, Abraham; Góra, Paweł, Laws of chaos. Invariant measures and dynamical systems in one dimension, Probability and its Applications, Birkhäuser Boston, Inc., Boston, MA, 1997, MR1461536 (99a:58102).

2. Boudourides, Moses A.; Fotiades, Nikos A., Piecewise linear interval maps both expanding and contracting, Dynamics and stability of systems 15 (2000), no. 4, 343-35, MR1809210 (2002a:37050)

3. Ding, Yi Ming; Fan, AI Hua; Yu, Jing Hu, Absolutely continuous invariant measures of piecewise linear lorenz maps, http://arxiv.org/pdf/1001.3014 
4. Dajani, Karma; Hartono, Yusuf; Kraaikamp, Cor, Mixing properties of $(\alpha, \beta)$-expansions, Ergod. Th. \& Dynam. Sys. (First published online 2009), 1-22.

5. Góra, Paweł, Invariant densities for piecewise linear maps of interval, Ergodic Th. and Dynamical Systems 29, Issue 05 (October 2009), 1549-1583.

6. Islam, Shafiqul, Absolutely continuous invariant measures of linear interval maps, Int. J. Pure Appl. Math. 27 (2006), no. 4, 449-464, MR2223985 (2006k:37100).

7. Lasota, A.; Yorke, James A., On the existence of invariant measures for piecewise monotonic transformations, Trans. Amer. Math. Soc. 186 (1973), 481-488, MR0335758 (49 \#538).

Department of Mathematics and Statistics, Concordia University, 1455 de Maisonneuve Blvd. West, Montreal, Quebec H3G 1M8, Canada

E-mail address: peyman_eslami@yahoo.com, pgora@mathstat.concordia.ca 